\title{
SIMILARITY OF QUADRATIC FORMS AND ISOMORPHISM OF THEIR FUNCTION FIELDS
}

\author{
BY
}

\section{ADRIAN R. WADSWORTH( $\left.{ }^{1}\right)$}

ABSTRACT. This paper considers the question: Given anisotropic quadratic forms $Q$ and $Q^{\prime}$ over a field $K$ (char $K \neq 2$ ), if their function fields are isomorphic must $Q$ and $Q^{\prime}$ be similar? It is proved that the answer is yes if $Q$ is a Pfister form or the pure part of a Pfister form, or a 4-dimensional form. The argument for $P$ fister forms and their pure parts does not generalize because these are the only anisotropic forms which attain maximal Witt index over their function fields. To handle the 4-dimensional case the following theorem is proved: If $Q$ and $Q^{\prime}$ are two 4-dimensional forms over $K$ with the same determinant $d$, then $Q$ and $Q^{\prime}$ are similar over $K$ iff they are similar over $K[\sqrt{d}]$. The example of Pfister neighbors suggests that quadratic forms arguments are unlikely to settle the original question for other kinds of forms.

Let $Q$ be a nonsingular quadratic form defined on an $n$-dimensional vector space $V(n \geq 3)$ over a field $K$ (char $K \neq 2)$, with diagonal representation $\left(a_{0}, a_{1}, \ldots, a_{n-1}\right)$. The function field of $Q, K_{Q}$, is the quotient field of $K\left[x_{1}, \ldots, x_{n-1}\right] /\left(a_{0}+a_{1} x_{1}^{2}+\cdots+a_{n-1} x_{n-1}^{2}\right)$, i.e., $K\left(x_{1}, \ldots, x_{n-2}\right)[\sqrt{-a}]$, where $a=a_{n-1}^{-1}\left(a_{0}+a_{1} x_{1}^{2}+\cdots+a_{n-2} x_{n-2}^{2}\right)$. The field $K_{Q}$ is uniquely determined (up to $K$-isomorphism) by $Q$, independent of the choice of diagonal representation. Further, if $Q^{\prime}$ is another quadratic form which is isometric to $Q$, or even similar to $Q$ (i.e., with a diagonal representation $\left(a a_{0}, a a_{1}\right.$, $\left.\ldots, a a_{n-1}\right)$ for some $\left.a \in K^{*}\right)$, then $K_{Q^{\prime}} \cong K_{Q^{*}}$

We consider here the converse of the last sentence:

(†) If $Q$ and $Q^{\prime}$ are two anisotropic forms over $K$, such that $K_{Q^{\prime}} \cong K_{Q^{\prime}}$ must $Q$ and $Q^{\prime}$ be similar?

We must confine attention exclusively to anisotropic forms when $\operatorname{dim} Q \geq 4$, since $K_{Q}$ is purely transcendental over $K$ iff $Q$ is isotropic.

Received by the editors April 23, 1974.

AMS (MOS) subject classifications (1970). Primary 15A63; Secondary 10C05.

Key words and phrases. Similar quadratic forms, Pfister form, function field.

(1) Much of the work presented he re was a part of my doctoral dissertation, which was prepared while I was an N.S.F. Graduate Fellow. I would like to thank my advisor, Irving Kaplansky, for suggesting this problem, and for many constructive conversations on it. 
It was proved long ago by Witt [7], using the theory of algebraic function fields in one variable, that the answer to $(t)$ is yes if the forms are threedimensional. In $\$ 1$ we will give an affirmative answer if $Q$ is a Pfister form or the pure part of a Pfister form (in particular, reproving Witt's result). In $\S 2$ we will show that the answer is again yes if $\operatorname{dim} Q=4$. We will indicate why the arguments used here do not generalize to other cases. Indeed, the example of Pfister neighbors suggests that quadratic-form-theoretic arguments will not settle $(\dagger)$ except in the cases analyzed here.( $\left.{ }^{2}\right)$

We begin with a few remarks on notation and terminology, and a standard lemma. Throughout the discussion the field $K$ will be fixed, with char $K \neq 2$. Field isomorphisms will be $K$-isomorphisms. Each quadratic form $Q$ will be nonsingular and (unless indicated otherwise) will be a $K$ form, i.e., a quadratic form defined on some finite-dimensional $K$-vector space $V$. As usual, $\operatorname{dim} Q$ is taken to be the dimension of $V$. The discriminant of $Q$, disc $Q=\bar{d}$, is the image in $K^{*} /\left(K^{*}\right)^{2}$ of the determinant $d$ of some matrix representing $Q$. (No sign is attached.) For $Q^{\prime}$ another $K$-form, $Q^{\prime} \cong Q$ means $Q$ and $Q^{\prime}$ are isometric. $Q^{\prime}$ is a subform of $Q$ if there is another $K$-form $Q^{\prime \prime}$ such that $Q \cong Q^{\prime} \perp Q^{\prime \prime}$. $Q \sim a$ means $Q$ represents $a$. For $a_{1}, a_{2}, \ldots \in K^{*},\left(a_{1}, \ldots, a_{n}\right)$ is an $n$-dimensional $K$-form which is represented by a diagonal matrix with entries $a_{1}, \ldots, a_{n}$. If $Q \cong$ $\left(a_{1}, \ldots, a_{n}\right)$, then $a Q \cong\left(a a_{1}, \ldots, a a_{n}\right), Q$ is nearly hyperbolic if it is the orthogonal sum of a hyperbolic form and a 1-dimensional form. If $L$ is a field containing $K, Q_{L}$ denotes the $L$-form defined on $V \otimes_{K} L$ induced by $Q$ on $V$. For standard quadratic form results quoted without reference, the reader is referred to [4] or [2].

A $P$ fister form $P$ is a $K$-form representable as a product $P \cong \bigotimes_{j=1}^{r}\left(1, b_{j}\right)$, for some $b_{1}, \ldots, b_{r} \in K^{*}$. Recall that for any $a \in K^{*}$, if $P \sim a$, then $P \cong a P$ and if $P$ is isotropic, then $P$ is hyperbolic. Of course, $P_{L}$ is again a Pfister form for any field $L \supseteq K$. Since $P \sim 1$ we have the decomposition $P \cong(1) \perp Q . Q$ is called the pure part of the Pfister form $P$. Observe that $Q_{L}$ is the pure part of $P_{L}$, and if $Q_{L}$ is isotropic, then it is nearly hyperbolic. A Pfister neighbor (following Knebusch's definition) is a quadratic form $R$ which is similar to a subform of a Pfister form $P$, with $\operatorname{dim} R>1 / 2 \operatorname{dim} P$. For details on Pfister forms, see Pfister's original paper [6], or the excellent accounts in [2] and [3].

We state for the reader's convenience a well-known lemma which pro-

(2) All of the results given here for the function field of a form apply equally well to its homogeneous function field, which is a simple purely transcendental extension of the function field. 
vides the opening wedge for our consideration of function fields. See, for example, $[2$, p. 200] for a proof.

Lemina 1. Take $d \in K^{*}$ and let $L=K[\sqrt{d}]$. Any anisotropic $K$-form $Q$ can be decomposed $Q \cong(1,-d) \otimes Q_{0} \perp Q_{1}$, where $Q_{1 L}$ is anisotropic. (In particular, if. $Q_{L}$ is hyperbolic, the $Q_{1}$ term disappears.)

1. Function fields of Pfister forms. The following notation will be used throughout this section. $Q$ will be a $K$-form of dimension $n \geq 3, m=n-2$. $F=K\left(x_{1}, \ldots, x_{m}\right)$ and $M=F\left(x_{m+1}\right)$, where the $x_{i}$ 's are independent indeterminates. Take a diagonal representation $\left(a_{0}, \ldots, a_{m}, a\right)$ of $Q$, and let $a=a^{-1}\left(a_{0}+a_{1} x_{1}^{2}+\cdots+a_{m} x_{m}^{2}\right)$ and $\beta=\alpha+x_{m+1}^{2}$. So $K_{Q} \cong F[\sqrt{-\alpha}]$.

Our first theorem collects the information needed to consider isomorphism of function fields of Pfister forms and their pure parts.

Theorem 2. Suppose $Q^{\prime}$ is an anisotropic quadratic form over K. Assume further (with $Q, \alpha, \beta$, as given above):

(a) $Q^{\prime}$ becomes hyperbolic in $K_{Q}$. Then,

(b) there is an F-form $Q_{0}$, such that $Q_{F}^{\prime} \cong(1, \alpha) \otimes Q_{0}$;

(c) $Q_{M}^{\prime} \cong \beta Q_{M}^{\prime}$;

(d) $Q$ is similar to a subform of $Q^{\prime}$. (Hence $\operatorname{dim} Q^{\prime} \geq \operatorname{dim}$ Q.)

Proof. We show $(a) \Rightarrow(b) \Rightarrow(c) \Rightarrow(d)$. Recall that the Witt index is preserved under purely transcendental field extensions. Thus, $Q_{F}^{\prime}$ is anisotropic. To obtain (b) from (a), apply Lemma 1 over $F$ with $d=-a$. Since $(1, \alpha)_{M} \sim \beta$, we have $(1, \alpha)_{M} \cong \beta(1, \alpha)_{M}$. (c) now follows at once. In particular, taking any $c \in K^{*}$ represented by $Q^{\prime}, Q_{M}^{\prime} \sim c \beta$. Applying the Cassels-Pfister subform theorem [6] (inhomogeneous form, but the same proof holds), it follows that $\left(c a^{-1} a_{0}, \ldots, c a^{-1} a_{m}, c\right)$ is a subform of $Q^{\prime}$. But this subform is just $c a^{-1} Q$, proving (d). Q.E.D.

Remark. (c) $\Rightarrow$ (a) holds as well. ( $(c) \Rightarrow$ (b) can be deduced by the Cassels-Pfister subform theorem.) Indeed, the equivalence of (a) and (c) is a special case of a beautiful theorem of Knebusch [1].

Theorem 3. If $Q^{\prime}$ is an anisotropic Pfister form $\left(\operatorname{dim} Q^{\prime} \geq 4\right)$ and $K_{Q} \cong K_{Q^{\prime}}$, then $Q$ and $Q^{\prime}$ are similar.

Proof. Being a Pfister form $Q^{\prime}$ becomes not merely isotropic, but actually hyperbolic in $K_{Q^{\prime}}$. Since $K_{Q} \cong K_{Q^{\prime}}$, Theorem 2 implies $Q$ is similar to a subform of $Q^{\prime}$. But $\operatorname{dim} Q=\operatorname{dim} Q^{\prime}$ because $K_{Q}$ and $K_{Q^{\prime}}$ have the same transcendence degree over $K$. So the subform must be all of $Q^{\prime}$, completing the proof. 
Theorem 4. If $Q^{\prime}$ is the pure part of an anisotropic Pfister form $\left(\operatorname{dim} Q^{\prime} \geq 3\right)$ and $K_{Q} \cong K_{Q^{\prime}}$, then $Q$ and $Q^{\prime}$ are similar.

Proof. Say $P \cong(1) \perp Q^{\prime}$, a Pfister form. Over $K_{Q}, Q^{\prime}$ becomes isotropic, so $P$ becomes hyperbolic. By Theorem 2, $Q$ is similar to a subform of $P$. But, by the transcendence degree argument just used, $\operatorname{dim} Q=\operatorname{dim} Q^{\prime}$. Thus, $P \cong c Q \perp(d)$ for some $c, d \in K^{*}$. Since $P \sim d, P \cong d P \cong c d Q \perp(1)$. By the Witt cancellation theorem, $Q^{\prime} \cong c d Q$. Q.E.D.

Note that the same kind of proof shows that if $Q^{\prime}$ is a Pfister neighbor of the anisotropic Pfister form $P$ and $K_{Q} \cong K_{Q^{\prime}}$, then $Q$ is also a Pfister neighbor of $P$ and $\operatorname{dim} Q=\operatorname{dim} Q^{\prime}$. (But see the comments at the end of $\S 2$.)

Since there is only one similarity class of isotropic 3 -dimensional forms, the condition that $Q^{\prime}$ be anisotropic can be deleted when $\operatorname{dim} Q^{\prime}=3$. Thus, we have reproved Witt's result which was the starting point of this investigation.

One might hope to generalize Theorems 3 and 4 to anisotropic forms which become hyperbolic or nearly hyperbolic over their function fields. The next two theorems show that there is no further generalization.(3)

Theorem 5. If $Q$ becomes hyperbolic over its function field $(\operatorname{dim} Q \geq 4)$, then $Q$ is already hyperbolic over $K$, or $Q$ is similar to an anisotropic $P$ fister form.

Proof. If $Q$ is isotropic, then $K_{Q}$ is purely transcendental over $K$. So $Q$ hyperbolic over $K_{Q}$. forces $Q$ hyperbolic over $K$.

Now assume $Q$ is anisotropic. Applying a suitable similarity factor, we may assume $Q \sim 1$. In the notation established above (with $a=1$ ), we take $Q^{\prime}=Q$, and Theorem 2 implies $Q_{M} \cong \beta Q_{M}$. That is, $Q$ is strongly multiplicative in the sense of Pfister [G] (inhomogeneous form). Being anisotropic, $Q$ must be a Pfister form. Q.E.D.

Theorem 6. If $Q$ becomes nearly hyperbolic over its function field $(\operatorname{dim} Q \geq 3)$, then $Q$ is already nearly hyperbolic over $K$, or $Q$ is similar to the pure part of an anisotropic Pfister form.

Proof. The isotropic case is handled just as in the preceding proof. So assume $Q$ is anisotropic. We simplify the notation by applying a similarity to obtain $Q \sim 1$ (which permits us to take $a=1$ in the expressions for $a$ and $\beta)$. Let $\bar{d}=\operatorname{disc} Q$.

Now, $Q_{F}$ is anisotropic, but $Q$ becomes nearly hyperbolic over $F[\sqrt{-\alpha}]=K_{Q}$. By Lemma $1, Q_{F} \cong(1, \alpha) \otimes Q_{0} \perp(\delta)$, for some $F$-form $Q_{0}$.

(3) Theorems 5 and 6 have been obtained independently by $\mathrm{K}_{\mathrm{n}}$ busch. 
( $\delta)$ can be determined by a comparison of discriminants. There are two possibilities, the first of which will be ruled out.

Case $1 . \operatorname{dim} Q \equiv 1(\bmod 4)$. Then $\operatorname{dim} Q_{0}$ is even, so that $\operatorname{disc}\left((1, a) \otimes Q_{0}\right)=\overline{1}$. Hence, we can take $\delta=d$, which shows that $Q_{F} \sim d$. However, as $F$ is purely transcendental over $K$, Cassel's theorem [3, p. 18] implies $Q \sim d$. This gives a decomposition of $Q$ over $K: Q \cong Q_{1} \perp(d)$. Cancelling $(d)_{F}$ from the two decompositions of $Q_{F}$ yields $Q_{1 F} \cong(1, a) \otimes$ $Q_{0}$. By Theorem $2((\mathrm{~b}) \Rightarrow(\mathrm{d})), Q$ must be similar to a subform of $Q_{1}$, which is absurd, as $\operatorname{dim} Q_{1}=\operatorname{dim} Q-1$. Thus, Case 1 can never occur.

Case 2. $\operatorname{dim} Q \equiv 3(\bmod 4)$. This time $\operatorname{dim} Q_{0}$ is odd, so that $\operatorname{disc}\left((1, \alpha) \otimes Q_{0}\right)=\bar{\alpha}$, and $(\delta) \cong(\alpha d)$. Let $P$ be the $K$-form $Q \perp(d)$, i.e., $P \cong\left(a_{0}, \ldots, a_{m}, 1, d\right)$. To complete the proof it suffices to show that $P$ is an anisotropic Pfister form. (Then $P \cong d P \cong(1) \perp d Q$. So $d Q$ is the pure part of $P$.)

The decomposition of $Q_{F}$ yields $P_{F} \cong(1, \alpha) \otimes\left(Q_{0} \perp(d)\right)$. So, by Theorem $2((\mathrm{~b}) \Rightarrow(\mathrm{c})), P_{M} \cong \beta P_{M}$. Hence, $P_{M}^{\prime} \cong \beta P_{M}^{\prime}$, where $P^{\prime}$ is the anisotropic part of $P$. By Theorem $2((\mathrm{c}) \Rightarrow(\mathrm{d})), Q$ is similar to a subform of $P^{\prime}$. Since $\operatorname{dim} Q=\operatorname{dim} P-1, P$ must be anisotropic.

Now take new independent indeterminates $y_{0}, \ldots, y_{n}$ and set $K^{\prime}=$ $K\left(y_{0}, \ldots, y_{n}\right)$ and $M^{\prime}=M\left(y_{0}, \ldots, y_{n}\right)=K^{\prime}\left(x_{1}, \ldots, x_{m+1}\right)$. Of course, $P_{M^{\prime}} \cong \beta P_{M^{\prime}}$. Take any $c \in K^{\prime}$, such that $P_{K^{\prime}} \sim c$. Then $P_{M^{\prime}} \sim c \beta$, so that, by the Cassels-Pfister subform theorem (treating $c \beta$ as a polynomial in the $x_{i}$ 's over $\left.K^{\prime}\right),\left(c a_{0}, c a_{1}, \ldots, c a_{m}, c\right)$ is a subform of $P_{K^{\prime}}$ A comparison of discriminants shows that $P_{K^{\prime}} \cong\left(c a_{0}, c a_{1}, \ldots, c, c d\right) \cong c P_{K^{\prime}}$. In particular, we may take $c=a_{0} y_{0}^{2}+a_{1} y_{1}^{2}+\cdots+a_{m} y_{m}^{2}+y_{m+1}^{2}+d y_{n}^{2}$, showing that $P$ is strongly multiplicative, hence a Pfister form. Q.E.D.

2. 4-dimensional forms. We now proceed to give an affirmative answer to question ( $\dagger$ ) for 4-dimensional forms. This is done by using the Pfister form results over a quadratic extension $L$ of $K$, then working back to $K$. The key step is the transition from $L$ to $K$, which is provided by the following theorem.

Theorem 7. Let $Q$ and $Q^{\prime}$ be two 4-dimensional K-forms, each representing 1 and each with discriminant $\bar{d}$. Let $L=K[\sqrt{d}]$. Then $Q$ and $Q^{\prime}$ are similar iff $Q_{L} \cong Q_{L}^{\prime}$.

Proof. Necessity. $Q_{L}$ and $Q_{L}^{\prime}$ are Pfister forms, and hence isometric whenever they are similar.

Sufficiency. Suppose $Q_{L} \cong Q_{L}^{\prime}$. Assume $d \notin\left(K^{*}\right)^{2}$-otherwise $L=K$ and there is nothing to prove. If $Q$ is isotropic, then so are $Q_{L}$ and $Q_{L}^{\prime}$, 
hence so is $Q^{\prime}$ (a well-known fact which can be deduced from Lemma 1 by a discriminant argument). But then disc $Q=\operatorname{disc} Q^{\prime}$ implies that $Q$ and $Q^{\prime}$ are similar. Thus, we may assume $Q$ and $Q^{\prime}$ are anisotropic.

Take decompositions $Q \cong(1) \perp R$ and $Q^{\prime} \cong(1) \perp R^{\prime}$, and let $S=R \perp$ $\left(-R^{\prime}\right)$. Since $Q_{L} \cong Q_{L}^{\prime}, S_{L}$ must be hyperbolic. If $S$ were anisotropic, Lemma 1 would force disc $S=-\bar{d}$. But $\operatorname{disc} S=\operatorname{disc} R \cdot\left(-\operatorname{disc} R^{\prime}\right)=$ $-\overline{1} \neq-\bar{d}$. Therefore, $S$ must be isotropic. Since $R$ and $R^{\prime}$ are anisotropic it follows that there is an $a \in K^{*}$, such that $R \sim a$ and $R^{\prime} \sim a$. Hence, there exist diagonal representations $Q \cong(1, a, b, b a d)$ and $Q^{\prime} \cong\left(1, a, b^{\prime}, b^{\prime} a d\right)$, for some $b, b^{\prime} \in K^{*}$.

We will demonstrate the similarity of $Q$ and $Q^{\prime}$ "piecewise", by finding a $t \in K^{*}$ with $(1, a) \cong t(1, a)$ and $\left(b^{\prime}, b^{\prime} a d\right) \cong t(b, b a d)$. To this end, consider the form $T=\left(1, a,-b b^{\prime},-b b^{\prime} a d\right)$, whose discriminant is $\bar{d}$. Working over $L$, where $d$ is a square, we have

$$
b T_{L} \cong\left(b, b a,-b^{\prime},-b^{\prime} a d\right)_{L} \cong\left(b, b a d,-b^{\prime},-b a d\right)_{L} .
$$

Thus, $b T_{L}$ is a subform of the hyperbolic form $Q_{L} \dot{L}-Q_{L}^{\prime}$, with complement $(1, a,-1,-a)_{L}$ which is also hyperbolic. Therefore, $T_{L}$ is hyperbolic. Using again the well-known fact quoted above, $T$ itself is isotropic. From a nontrivial representation of 0 by $T$, we obtain $t \in K^{*}$, such that $(1, a) \sim t$ and $\left(b b^{\prime}, b b^{\prime} a d\right) \sim t$. The two piecewise similarities now follow at once. Thus, $Q^{\prime} \sim t Q$. Q.E.D.

This theorem may be of some interest in itsown right. One immediate consequence is that a Hasse principle for similarity of 4-dimensional forms over a global field may be deduced from the Hasse principle for isometry. (4) (The global square theorem $[4,65: 15]$ shows that equality of discriminants can be verified locally.)

For the proof of our final theorem, it is convenient to recast Theorem 7 as follows: If $Q$ and $Q^{\prime}$ are 4-dimensional forms each with discriminant $\bar{d}$ and $L=K[\sqrt{d}]$, then $Q$ and $Q^{\prime}$ are similar iff $Q_{L}$ and $Q_{L}^{\prime}$ are similar.

Theorem 8. Let $Q$ and $Q^{\prime}$ be anisotropic 4-dimensional forms with $K_{Q} \cong K_{Q^{\prime \prime}}$. Then $Q$ and $Q^{\prime}$ are similar.

Proof. Let $\bar{d}=\operatorname{disc} Q$ and $\overline{d^{\prime}}=\operatorname{disc} Q^{\prime}$. If $\bar{d}=\overline{1}$, then $Q$ is similar to an anisotropic Pfister form (with the same function field), and Theorem 3 applies. Therefore, we may assume that $d, d^{\prime} \notin\left(K^{*}\right)^{2}$.

Let $L=K[\sqrt{d}]$. Note that because $K_{Q}$ is a regular extension of $K$, the $K$-isomorphism of $K_{Q}$ and $K_{Q^{\prime}}$ induces an $L$-isomorphism of $L_{Q_{L}}$ of $L_{Q_{L}^{\prime}}$.

(4) Ono [5] has shown that the Hasse principle for similarity holds for forms of any dimension over a global field. 
By Theorem 3 ( $Q_{L}$ being similar to a Pfister form), $Q_{L}$ and $Q_{L}^{\prime}$ are similar. In particular, disc $Q_{L}=\operatorname{disc} Q_{L}^{\prime}$, i.e., $d^{\prime} \in\left(L^{*}\right)^{2}$. Since $d^{\prime} \in K^{*}-\left(K^{*}\right)^{2}$, an easy computation (or an application of Lemma 1 to $\left(1,-d^{\prime}\right)$ ) shows that $\overline{d^{\prime}}=\bar{d}$. Thus, we may apply Theorem 7 (as recast) to conclude that $Q$ and $Q^{\prime}$ are similar. Q.E.D.

The techniques used for Pfister forms and 4-dimensional forms do not generalize readily to other kinds of forms. To illustrate the problems that can be encountered, it suffices to consider the example of Pfister neighbors. Let $Q$ and $Q^{\prime}$ be two dissimilar forms of the same dimension, which are each a Pfister neighbor of the same anisotropic Pfister form $P$. (So $\operatorname{dim} P=2^{r}$, for $r \geq 3$, and $2^{r-1}<\operatorname{dim} Q<2^{r}-1$.) Then in any extension field $F$ of $K, Q_{F}$ is isotropic iff $P_{F}$ is hyperbolic iff $Q_{F}^{\prime}$ is isotropic. In particular, $Q$ is isotropic over $K_{Q^{\prime}}$, and vice versa. (In fact, it can be shown that for any $K$-form $R, R$ is isotropic (resp. hyperbolic) over $K_{Q}$ iff $R$ is isotropic (resp. hyperbolic) over $K_{Q^{\prime}}$. ) Further, for every algebraic extension $L$ of $K$, $L_{Q_{L}}$ is purely transcendental over $L$ iff $L_{Q_{L}^{\prime}}$ is purely transcendental over $L$. Whether $K_{Q}$ and $K_{Q^{\prime}}$ can be isomorphic is unclear. It appears that new techniques will be necessary to settle this question.

Added in proof. M. Knebusch has shown that dissimilar Pfister neighbors often have isomorphic function fields. Specifically, if $Q$ and $Q^{\prime}$ are neighbors of the Pfister form $P$, with $\operatorname{dim} P=2^{r}$, and if $Q$ and $Q^{\prime}$ each have subforms similar to the Pfister form $R$, with $\operatorname{dim} R=2^{r-1}$, and $\operatorname{dim} Q=$ $\operatorname{dim} Q^{\prime}$, then $K_{Q} \cong K_{Q^{\prime}}$. This is one of many interesting results in his excellent paper Generic splitting of quadratic forms. I, to appear in Proc. London Math. Soc.

\section{REFERENCES}

1. M. Knebusch, Specialization of quadratic and symmetric bilinear forms, and a norm theorem, Acta Math. 24 (1973), 279-299.

2. T. Y. Lam, The algebraic theory of quadratic forms, Benjamin, Reading, Mass., 1973.

3. F. Lorenz, Quadratische Formen über Körpern, Lecture Notes in Math., vol. 130 , Springer-Verlag, Berlin and New York, 1970. MR $44 \# 189$.

4. O. T. O'Meara, Introduction to quadratic forms, Die Grundlehren der Math. Wissenschaften, Band 117, Academic Press, New York; Springer-Verlag, Berlin, 1963. MR 27 \#2485.

5. T. Ono, Arithmetic of orthogonal groups, J. Math. Soc. Japan 7(1955), 79-91. MR 16, 1087.

6. A. Pfister, Multiplicative quadratische Formen, Arch. Math. 16 (1965), 363-370. MR $32 \# 2408$.

7. E. Witt, Uber ein Gegenbeispiel zum Normensatz, Math. Z. 39 (1935), 462-467.

DEPARTMENT OF MATHEMATICS, UNIVERSITY OF CALIFORNIA, BERKELEY, CALIFORNIA 94720

Current address: Department of Mathematics, University of California at San Diego, La Jolla, California 92037 